concentric diminution of the visual field is said to be due to involvement of the central portion of the nerve. In the first place a central scotoma is very rare in neuro-syphilis, and there is considerable doubt as to whether its occurrence as the result of syphilis has ever been satisfactorily proved. Usually the visual failure is either concentric starting peripherally or a uniform loss starting all over the field at the same time. The macular fibres, though lying on the lateral side of the optic nerve close to the eye, soon come to occupy a central position.

On the question of prognosis in parenchymatous neuro-syphilis, Dr. Worster-Drought appears to be unduly pessimistic, which can be explained by the fact that one of his criteria of diagnosis between meningo-vascular and parenchymatous neurosyphilis is that the latter does not respond to ordinary anti-syphilitic remedies or to Tryparsamide injections. While this may be true of "ordinary" anti-syphilitic remedies (tri-valent arsphenamines), it is demonstrably untrue in the case of Tryparsamide, by the use of which in adequate amounts complete remission has been obtained in significant numbers of cases of dementia paralytica. Again the mental symptoms of cerebral interstitial syphilis can with difficulty be distinguished from those of dementia paralytica, and dependence is placed by the author mainly on the length of time between the original infection and the development of symptoms and on the response to treatment for his diagnosis. Unfortunately an accurate statement of the date of the initial infection is usually difficult to obtain, if indeed a history of infection can be obtained at all. The limits between which dementia paralytica may develop are set at 5-20 years from the infection, whereas incubation periods of 40 and even 50 years have been observed.

On therapy the book is less illuminating and authoritative than on pure neurology. As regards malaria, the author prefers infection by blood injection rather than by mosquito bite, the usual preference being the reverse of this. The suggestion on page 139 that a second course of malaria may be given after one year, in cases of dementia paralytica which fail to show improvement from the first course, must be called in question. A patient who does not respond by improvement in mental symptoms after one adequate course of malaria will never do so, and a second attempt is contra-indicated. The only valid indication for a second course after an interval of $1-2$ years is the persistence of clinical or serological evidence of dementia paralytica in a patient who has given a satisfactory response to the first course. Tryparsamide, though of proved value in parenchymatous neuro-syphilis, receives scant mention and little recommendation, as do other methods of inducing satisfactory and controllable fever. It is probable that pyrexia produced by physical means (Electropyrexia, the Kettering Hypertherm, etc.) will eventually come to hold the field. American opinion is definitely that the therapeutic effect is due solely to adequate pyrexia for an adequate period and that malaria has no specific effect in dementia paralytica other than as a pyrexial agent. Injections of non-specific vaccines and of sulphur as pyrexial agents are inadequate and have disappeared from general use. On page 97, the strength of Vivatol is given as the original strength of the preparation, which has for some time now been doubled.

The index is good and the bibliography extensive, although in many instances ancient history.

\section{ROSS AND FAIRLIE'S HANDBOOK OF ANÆSTHETICS.}

Revised by R. J. Minnitr, M.D. Fifth Edition. E. \& S. Livingstone, Edinburgh. 1940. Price 12s. 6d.

Dr. Minnitt has undertaken to revise Ross and Fairlie's Handbook of Anæsthetics with great care, and has devoted an immense amount of time to this work.

Three new chapters have been added, which deal with cyclopropane, closed circuit absorption methods, vinesthene, and the therapeutic use of oxygen helium and carbon dioxide.

These chapters are well written and easy to understand.

The student will find the chapters on the absorption and physiological action of anæsthetic drugs very well put, as is also the physiology of the respiratory system.

Chloroform and ether anæsthesia are adequately dealt with, as is the chapter on endotracheal anæsthesia, but as for some of the illustrations and descriptions of apparatus-now out of date-the reviewer feels that Dr. Minnitt has been too mild in his revision, and that it would have been much better to have cut them out. The chapters on the posture of the patient and accidents of anæsthesia are good and most helpful. The anæsthetist should know the postures required by the surgeon, and be able to realise the importance this will have during anæsthesia.

A word on the importance of conferring with the surgeon before the administration of the anæsthetic would not be out of place. The chapter on the choice of anæsthesia could be improved, and here again the writer has tried to be too loyal to the original work. Analgesia and anæsthesia in obstetrics are very well discussed as one would expect from one who has done so much work in this field.

The section on local anæsthesia is fairly well described, but perhaps it could have done with a little more detail.

Spinal anæsthesia is described, but here there have been some omissions, as for instance, the technique of Etherington Wilson, which is a modification of the Howard Jones method, and there is no mention of heavy percaine. It is good to see planocane advocated as a spinal agent. It is one that has been well tried and is safe.

Dr. Minnitt bids fair to make Ross \& Fairlie a much better and more popular book among students in London than formerly. It is of a convenient size, well printed and easy to read.

\section{EARLY TREATMENT OF NERVOUS AND MENTAL DISORDERS.}

By W. Lindesay Neustatter, M.R.C.P. 370 pp. J. \& A. Churchill, Ltd., London. 1940. Price 15s.

This is a new book and the author sets out, as he says, to try and bridge the gap between the general practitioner, the psychotherapist and the mental hospital psychiatrist. Those of us who have lived to years of greater discretion would have been severely daunted by this task, and perhaps it is one of the greatest attractions of the book that Dr. Neustatter has set about his task in a gay and debonair spirit and has tackled it 